\title{
Causal association of type 2 diabetes with amyotrophic lateral sclerosis: new evidence from Mendelian randomization using GWAS summary statistics
}

Ping Zeng ${ }^{1 *}$ (D) Ting Wang ${ }^{1}$, Junnian Zheng ${ }^{2,3,4^{*}}$ and Xiang Zhou ${ }^{5,6^{*}}$

\begin{abstract}
Background: Associations between type 2 diabetes (T2D) and amyotrophic lateral sclerosis (ALS) were discovered in observational studies in both European and East Asian populations. However, whether such associations are causal remains largely unknown.

Methods: We employed a two-sample Mendelian randomization approach to evaluate the causal relationship of T2D with the risk of ALS in both European and East Asian populations. Our analysis was implemented using summary statistics obtained from large-scale genome-wide association studies with 660,000 individuals for T2D and $\sim 81,000$ individuals for ALS in the European population, and 191,000 individuals for T2D and 4100 individuals for ALS in the East Asian population. The causal relationship between T2D and ALS in both populations was estimated using the inverse-variance-weighted methods and was further validated through extensive complementary and sensitivity analyses.
\end{abstract}

Results: Using multiple instruments that were strongly associated with T2D, a negative association between T2D and ALS was identified in the European population with the odds ratio (OR) estimated to be 0.93 ( $95 \% \mathrm{Cl} 0.88-0.99, p=$ 0.023), while a positive association between T2D and ALS was observed in the East Asian population with $\mathrm{OR}=1.28$ (95\% Cl 0.99-1.62, $p=0.058)$. These results were robust against instrument selection, various modeling misspecifications, and estimation biases, with the Egger regression and MR-PRESSO ruling out the possibility of horizontal pleiotropic effects of instruments. However, no causal association was found between T2Drelated exposures (including glycemic traits) and ALS in the European population.

Conclusion: Our results provide new evidence supporting the causal neuroprotective role of T2D on ALS in the European population and provide empirically suggestive evidence of increasing risk of T2D on ALS in the East Asian population. Our results have an important implication on ALS pathology, paving ways for developing therapeutic strategies across multiple populations.

Keywords: Amyotrophic lateral sclerosis, Type 2 diabetes, Two-sample Mendelian randomization, Instrumental variable, Genome-wide association studies, East Asian, European

\footnotetext{
* Correspondence: zpstat@xzhmu.edu.cn; jnzheng@xzhmu.edu.cn;

xzhousph@umich.edu

${ }^{1}$ Department of Epidemiology and Biostatistics, School of Public Health,

Xuzhou Medical University, Xuzhou 221004, Jiangsu, China

${ }^{2}$ Cancer Institute, Xuzhou Medical University, Xuzhou 221004, Jiangsu, China

${ }^{5}$ Department of Biostatistics, University of Michigan, Ann Arbor, Ml 48109,

USA

Full list of author information is available at the end of the article
}

(c) The Author(s). 2019 Open Access This article is distributed under the terms of the Creative Commons Attribution 4.0 International License (http://creativecommons.org/licenses/by/4.0/), which permits unrestricted use, distribution, and reproduction in any medium, provided you give appropriate credit to the original author(s) and the source, provide a link to the Creative Commons license, and indicate if changes were made. The Creative Commons Public Domain Dedication waiver (http://creativecommons.org/publicdomain/zero/1.0/) applies to the data made available in this article, unless otherwise stated. 


\section{Background}

Amyotrophic lateral sclerosis (ALS) is an adult-onset neurodegenerative disease characterized by rapid motor neuron degeneration and subsequent respiratory failure [1]. ALS is relatively rare worldwide: the standardized disease incidence is about 1.89 and 0.83 per 100,000 person-years of follow-up in the European and East Asian populations, respectively [2]. However, the burden of ALS is substantial. Clinically, ALS patients often suffer from loss of independence due to progressive functional impairments of upper and lower motor neurons. In addition, ALS patients only have a median survival time of 2-4 years starting from disease onset and less than $10 \%$ patients can survive beyond 10 years [3]. Economically, the annual cost per ALS patient is considerable: it ranges from $\sim \$ 11,000$ in Denmark to $\sim \$ 70,000$ in the USA [4]. Subsequently, the population-wide national economic burden of ALS is estimated to be $\sim \$ 1.023$ billion in the USA, far greater than that of the other two common neuromuscular diseases (Duchenne muscular dystrophy and myotonic dystrophy) [5]. Importantly, ALS incidence is expected to increase due to population aging worldwide, further aggravating the socioeconomic burden associated with ALS in the coming years [6]. Therefore, it becomes critically important to understand the etiology of ALS and identify risk factors that can causally influence ALS. Identifying causal risk factors for ALS can potentially lead to the discovery of new pathogenic pathways underlying ALS, guide the development of effective medical treatment and patient care, and facilitate healthcare policy making and healthcare resource allocation.

While the causes and pathogenesis of ALS remain largely unknown, several genetic and environmental risk factors have been identified to be associated with the development of ALS [7-9]. Among these identified risk factors, the association between antecedent diseases and ALS is of particular interest. Specifically, previous observational epidemiological studies have found that ALS patients tend to have less antecedent diseases (e.g., liver/ lung/thyroid disease, diabetes, hypertension, hyperlipidemia, and arthritis) as compared to the general age/gender/geography-matched control patients [10-13]. In addition, the presence of antecedent diseases appears to lead to a substantial delay in the onset age of ALS patients [10-13]. The evidence suggests that antecedent diseases may be markers of causal risk factors for ALS or may themselves be involved in the pathogenesis of ALS. Importantly, several pieces of evidence have recently emerged to support a potentially pathological role of one antecedent metabolic disease, type 2 diabetes (T2D), in ALS. The association between T2D and ALS was first discovered a decade ago [12]. Follow-up observational studies and hospital disease registries have provided additional empirical evidence supporting the association between T2D and ALS (Table 1). This association evidence, when further paired with the observations that high energy consumption often accompanies ALS progression [23, 24] and the observations that glucose intolerance and insulin resistance have been linked to ALS [25, 26], leads to a hypothesis that T2D may mechanically and causally affect ALS.

Unfortunately, establishing a causal relationship between T2D and ALS has been challenging so far. Almost all previous studies on T2D and ALS are observational in nature, and observational studies have inherent drawbacks that make it hard for them to reach a causal conclusion using standard statistical tools. In particular, while these previous observational studies have attempted to adjust for the effects of many confounding factors (e.g., income, education, marital status, age, gender, and race) (Additional file 1: Table S1), it is not possible to control for all confounding factors there. Unadjusted confounding factors can potentially bias the association evidence between T2D and ALS. Moving beyond observational studies is not straightforward either. For example, because ALS is rare in the population, it becomes difficult to collect large samples to carry out longitudinal studies for examining the influence of T2D on ALS [27]. In addition, due to ethical considerations, it is almost impossible to validate the causal association between T2D and ALS directly by performing randomized controlled trails. Therefore, it remains unclear whether the association between T2D and ALS observed in previous studies is causal or not: is T2D

Table 1 Estimated effect sizes of T2D on ALS in previous observational studies

\begin{tabular}{lllll}
\hline First author & Year & Nation & Effect size $(95 \%$ Cl) & Ref \\
\hline DOvidio & 2018 & Italy & $0.30(0.19-0.45)$ & {$[14]$} \\
Visser & 2017 & Netherlands & $0.77(0.33-1.21)$ & {$[15]$} \\
Hollinger & 2016 & USA & $0.80(0.53-1.21)$ & {$[10]$} \\
Mitchell & 2015 & USA & $0.47(0.38-0.58)$ & {$[11]$} \\
Mariosa & 2015 & Sweden & $0.79(0.68-0.91)$ & {$[16]$} \\
Seelen & 2014 & Netherlands & $0.72(0.51-1.01)$ & {$[17]$} \\
Turner & 2013 & England & $0.98(0.85-1.13)$ & {$[18]$} \\
Kioumourtzoglou & 2015 & Denmark & $0.61(0.46-0.80)$ & {$[19]$} \\
Moglia & 2017 & Italy & $1.05(0.78-1.42)$ & {$[20]$} \\
Korner & 2012 & Germany & $1.11(0.76-1.60)$ & {$[21]$} \\
Armon & 1991 & USA & $1.00(0.29-3.50)$ & {$[13]$} \\
Sun & 2015 & China & $1.35(1.10-1.67)$ & {$[22]$} \\
Pool 1 & & & $0.73(0.59-0.90)$ & \\
Pool 2 & & & $0.77(0.62-0.96)$ & \\
\hline
\end{tabular}

Pool 1: the effect size estimated without the study of Sun [22] as it was performed on the East Asian population; pool 2: the effect size estimated with all the studies; both estimations were generated by random-effects meta-analysis models 
protective against ALS or is the absence of T2D just an early manifestation of ALS [10, 11, 14, 19, 28, 29]?

Besides a lack of evidence on the causal relationship between T2D and ALS, likely also due to the observational nature of previous studies, there is a lack of consensus on whether T2D is protective for ALS in all human populations. For example, in the European population, despite minor conflicting results, most observational studies found that T2D is associated with decreased susceptibility to ALS (Additional file 1: Figure S1), suggesting a possible neuroprotection role of T2D on ALS. In contrast, however, in the East Asian population, it was found that T2D can increase the risk of ALS [22].

Mendelian randomization (MR) is an advanced statistical method that can help establish a causal relationship between an exposure of interest (e.g., T2D in the present study) and an outcome of interest in observational studies by employing single-nucleotide polymorphisms (SNPs) as instrumental variables for the exposure [3036]. MR relies on the idea that SNPs associated with T2D would also be associated with the risk of ALS through the path of T2D, if T2D is causally associated with ALS. Therefore, even though SNPs that are selected as instruments are not causal for T2D but are only associated with T2D, MR can still help establish the causal association between T2D and ALS [37]. Large-scale genome-wide association studies (GWASs) on T2D performed in the recent years have identified many SNPs associated with T2D, making it feasible to choose appropriate SNPs to serve as valid instruments for T2D [38].

To ensure the validity of the causal conclusion from MR, each selected instrumental variable needs to satisfy three MR modeling assumptions (Additional file 1: Figure S2A and Additional file 2) [39-41]: (i) it should be strongly associated with T2D; this is referred to as the relevance assumption; (ii) it should not be associated with any other confounders that may be associated with both T2D and ALS; this is referred to as the independence assumption; (iii) it influences ALS only through the path of T2D and does not have horizontal pleiotropic effects; this is referred to as the exclusion restriction assumption. Note that the first assumption (i.e., the relevance assumption) can be directly tested based on the observed data while the last two assumptions (i.e., the independence and exclusion restriction assumptions) are difficult to validate in practice. We will later examine the validity of the last two assumptions through various sensitivity analyses.

In the present study, our main objective is to investigate the causal relationship between T2D and ALS in both the European and East Asian populations. To achieve this objective, we conducted the largest twosample MR analysis to date based on summary statistics publicly available from large-scale GWASs with $\sim 63,000$ cases for T2D and $\sim 42,000$ cases for ALS in the European population, and with $\sim 191,000$ individuals for T2D and 4100 individuals for ALS in the East Asian population.

\section{Methods \\ GWAS data sources and instrument selection}

A crucial step of MR is to choose appropriate genetic variants to serve as valid instrumental variables for T2D. To do so, we obtained association results from one of the largest T2D GWASs to date [38], which was a genome-wide meta-analysis on three previous T2D studies: DIAbetes Genetics Replication and Meta-analysis (DIAGRAM) [42], Genetic Epidemiology Research on Adult Health and Aging (GERA) [43], and UK Biobank cohort [44]. Together, the T2D GWAS contained a total of $\sim 16$ million genotyped and imputed SNPs for 659,316 $(62,892$ T2D cases and 596,424 controls) individuals of European ancestry. Based on this GWAS data set, we followed other previous MR studies (e.g., [41, 45]) with a stringent selection procedure as described in Fig. 1. Specifically, we obtained a total of 139 index SNPs for T2D as candidate instrumental variables. We removed SNPs that have potential pleiotropic associations with ALS (defined by an ALS association $p$ value below the genome-wide suggestive significance level of 1.00E-5). We also excluded lipid-associated index SNPs as blood lipid levels have been shown to be associated with both T2D and ALS $[45,52]$. Note that the removal of pleiotropic SNPs is a conservative strategy and doing so can often ensure the validity of Mendelian randomization analysis with more confidence [33, 34, 45, 55-57]. However, to avoid the concern of excluding too many SNPs with potentially vertical pleiotropic effects $[58,59]$, we also performed sensitivity analysis with all 139 index SNPs included. Finally, we kept a total of 67 independent index SNPs $(p<5.00 \mathrm{E}-8)$ to serve as instrumental variables for T2D (Table 2). We obtained summary statistics (e.g., marginal effect sizes, their standard error, and effect allele) of these index SNPs for T2D from http://cnsgenomics.com/data.html. In addition, we also obtained association results from the largest ALS GWAS to date: a case-control study that analyzed $\sim 10$ million genotyped and imputed SNPs on 80,610 individuals (20, 806 ALS cases and 59,804 controls) of European ancestry [49]. We obtained the summary statistics of ALS for the same set of instrument variables from the ALS Variant Server [49].

While we primarily focused on examining the causal association between T2D and ALS, we also attempted to perform MR to estimate the causal effects of multiple T2D-related glycemic/anthropometric traits on ALS in the European population. These traits include fasting 


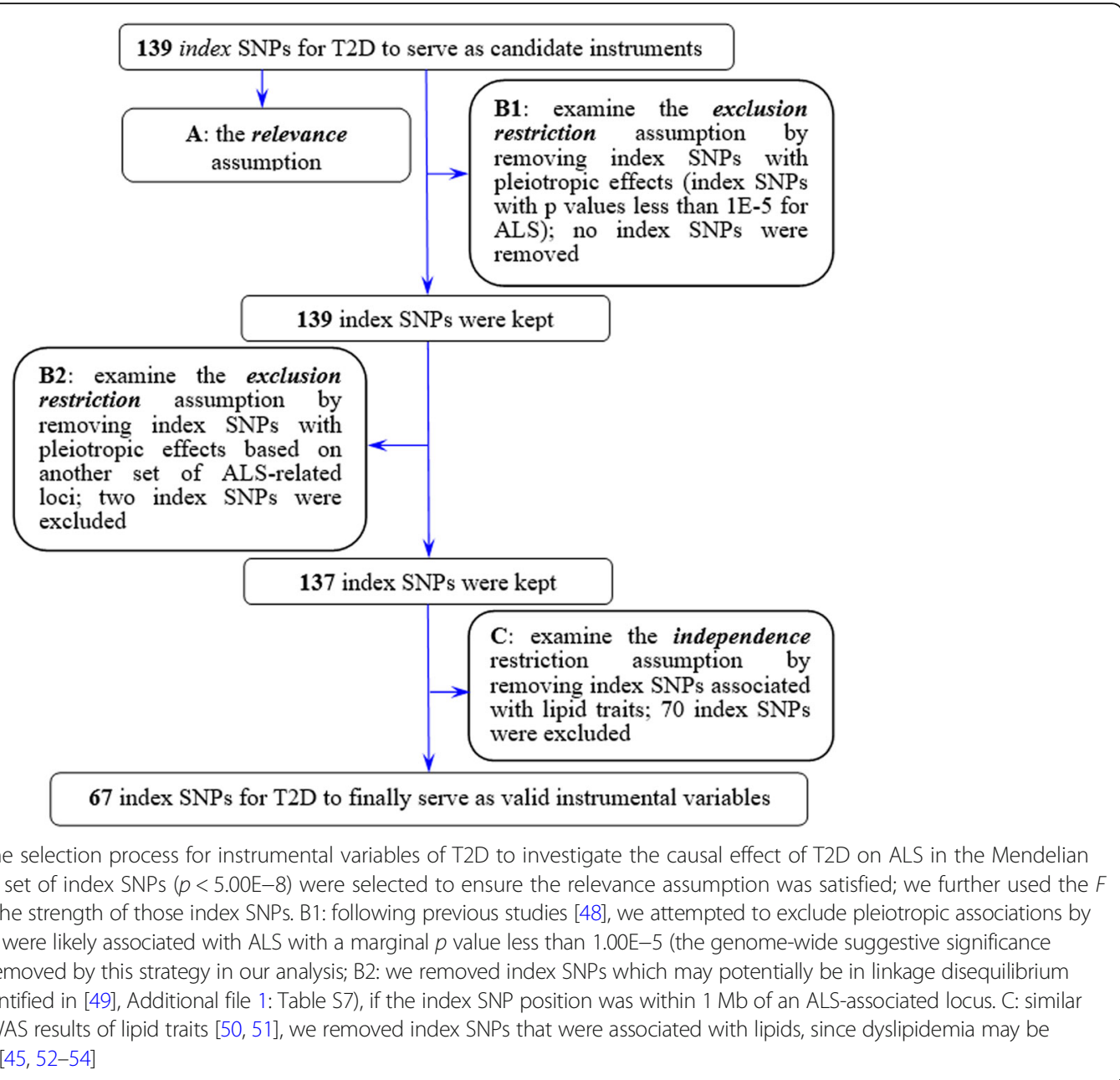

Table 2 GWAS data sets used in the present MR analysis

\begin{tabular}{|c|c|c|c|c|}
\hline Traits & Pop & $k_{1} / k_{0}$ & Sample size (case/control) & Data source \\
\hline ALS & EUR & & $20,806 / 59,804$ & AVS [49] \\
\hline $\mathrm{T} 2 \mathrm{D}$ & EUR & $67 / 139$ & $62,892 / 596,424$ & PCTG [38] \\
\hline $\mathrm{BMI}$ & EUR & $91 / 95$ & 339,224 & GIANT [60] \\
\hline Fasting glucose & EUR & $35 / 35$ & 133,010 & MAGIC [61] \\
\hline Fasting insulin & EUR & $14 / 14$ & 108,557 & MAGIC [61] \\
\hline $\mathrm{HbA1c}$ & EUR & $36 / 37$ & 123,665 & MAGIC [62] \\
\hline ALS & EAS & & $1234 / 2850$ & Benyamin [63] \\
\hline $\mathrm{T} 2 \mathrm{D}$ & EAS & $34 / 72$ & $36,614 / 155,150$ & JENGER [64] \\
\hline
\end{tabular}

Here $k_{1}$ is the final number of instruments employed in the analysis while $k_{0}$ is the number of candidate instruments. For T2D, we conducted stringent procedures (Fig. 1) to carefully choose instruments. For the remaining exposures, we performed the standard B1 and B2 selection procedures shown in Fig. 1 to select the final set of instrumental variables

T2D type 2 diabetes, BMI body mass index, ALS amyotrophic lateral sclerosis, HbA1C hemoglobin A1c, GIANT Genetic Investigation of ANthropometric Traits Consortium, MAGIC Meta-Analyses of Glucose and Insulin-related traits Consortium, JENGER Japanese ENcyclopedia of GEnetic associations by Riken, CHN China Health and Nutrition Survey, AVS ALS Variant Server, PCTG Program in Complex Trait Genomics, EUR European, EAS East Asian 
glucose [61], fasting insulin [61], hemoglobin A1c (HbA1c) [62], and body mass index (BMI) [60]. Using a selection procedure similar to that shown above, we obtained the SNP instrumental variables (Table 2) from the corresponding GWASs (Additional file 1: Tables S2S6). The GWAS genetic data sets employed in the present study are summarized in Table 2, with more details shown in Additional file 3.

\section{Complementary and statistical analysis}

For each index SNP that was selected as an instrument variable, we first quantified whether it was strongly associated with the exposure (e.g., T2D) or not. To do so, we calculated the phenotypic variance for the exposure variable explained (in the observed scale) by an individual instrument following the method in [65] and then computed the $F$ statistic [46, 47]. Afterwards, we carried out the two-sample MR to estimate the causal effect of T2D on ALS by applying both fixed-effects and random-effects inverse-variance-weighted (IVW) methods (see Additional file 2 for more descriptions on MR estimation methods) [47, 66-68]. We also created informative plots (e.g., SNP effect scatter and funnel plots) to illustrate the results.

Besides the above main analysis, we evaluated for potential violations of the model assumptions in the MR analysis by performing a number of complementary sensitivity analyses: (i) leave-one-out (LOO) cross-validation analysis [48] and Mendelian Randomization Pleiotropy RESidual Sum and Outlier (MR-PRESSO) analysis [69] for outlier instrument detection; (ii) weighted medianbased method for examining result robustness when some instruments may be potentially invalid [70]; (iii) MR-Egger regression, where its intercept was used to evaluate the directional pleiotropy of instruments [67, 71]. To further exclude bias due to horizontal pleiotropy, we also searched PhenoScanner [72] and the NHGRIEBI GWAS Catalog to examine whether some of the selected instruments may have association with other diseases or traits. We found that 17 instrumental variables were associated with some diseases or traits (e.g., blood pressure and sugar levels) in these databases (Additional file 1: Table S7). To avoid estimation bias due to these pleiotropic associations, we excluded these 17 instruments and carried out another IVW analysis to estimate the causal effect of T2D on ALS: (iv) reverse causal inference on T2D using ALS instrument variables to explore whether ALS has a causal impact on T2D (Additional file 1: Table S8); (v) IVW methods for estimating the causal effect of T2D [64] on ALS [63] in East Asians, where both the T2D and ALS GWAS summary statistics were obtained from East Asian individuals (Table 2 and Additional file 1: Table S9).

We matched the effect/alternative allele of instrument variables between exposures (e.g., T2D) and ALS during the analysis. The main statistical analyses were conducted within the $\mathrm{R}$ (version 3.5.2) environment for statistical computing. The statistical significance level was set to 0.05 throughout our study. Note that participants had given informed consent for data sharing as described in each of the original GWASs employed in the present manuscript. Therefore, additional ethical review was not needed for our study.

\section{Results}

\section{Causal effect of T2D on ALS}

A total of 67 instrumental variables of T2D were carefully selected (Fig. 1). All the selected instruments together explain about $0.61 \%$ phenotypic variation of $\mathrm{T} 2 \mathrm{D}$ at the observed scale (Additional file 1: Table S2). For these instrumental variables, all the $F$ statistics are above 10 (ranging from 29.8 to 251.0 ) with an average $F$ statistic of 59.8 and an overall $F$ statistic of 60.2 (Additional file 1: Table S2), indicating that they satisfy the strong relevance assumption of MR and that the weak instrument bias would not substantially influence the estimations of causal effects.

Combining all the instruments together, we found that T2D is negatively associated with ALS. Specifically, in terms of the fixed-effects IVW method, the odds ratio (OR) of T2D on ALS is estimated to be 0.93 (95\% CI $0.88-0.99, p=0.023)$, suggesting that being in T2D status can lead to an average of $6.57 \%$ (95\% CI $0.01-11.8 \%$ ) reduction in the risk of ALS (Fig. 2a). This inverse association suggests that T2D may play a causal neuroprotective role on the development of ALS. The randomeffects IVW method yields similar results, with a slightly conservative confidence interval $(\mathrm{OR}=0.93,95 \% \mathrm{CI}$ $0.87-1.00, p=0.055)$ due to the higher estimation variation resulting from considering the instrumental heterogeneity into model fitting. Using all the original 139 T2D instrumental variables, we obtained a similar negative causal effect of T2D on ALS using the randomeffects IVW method $(\mathrm{OR}=0.96$, 95\% CI 0.92-1.00, $p=$ 0.046), supporting the robustness of the main results. This inverse association can also be observed when another set of 90 T2D instruments obtained from a separate study was used (description of the data is provided in Additional file 3; results are shown in Additional file 1: Table S10), further supporting our results.

Besides estimating the causal effect of T2D on ALS, we also evaluated the causal effects of four T2D-related glycemic/anthropometric traits (BMI, fasting glucose, fasting insulin, and HbA1c) on ALS in the European population using IVW methods. However, no statistically significant causal associations are identified between each of those exposures and ALS. Specifically, in terms of the random-effects IVW method, the ORs for a unit change of BMI (where one unit equals $\sim 4.8 \mathrm{~kg} / \mathrm{m}^{2}$ ), 

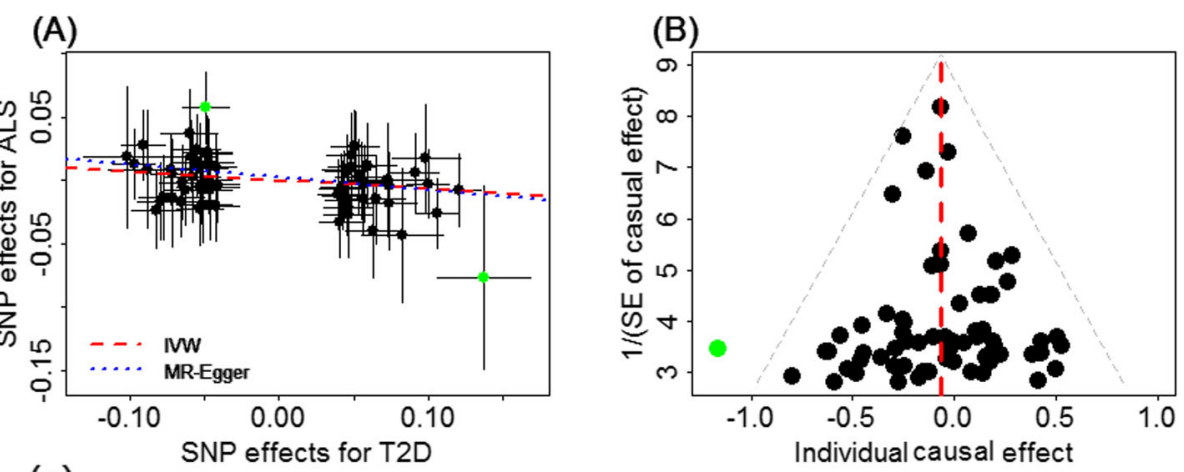

(c)

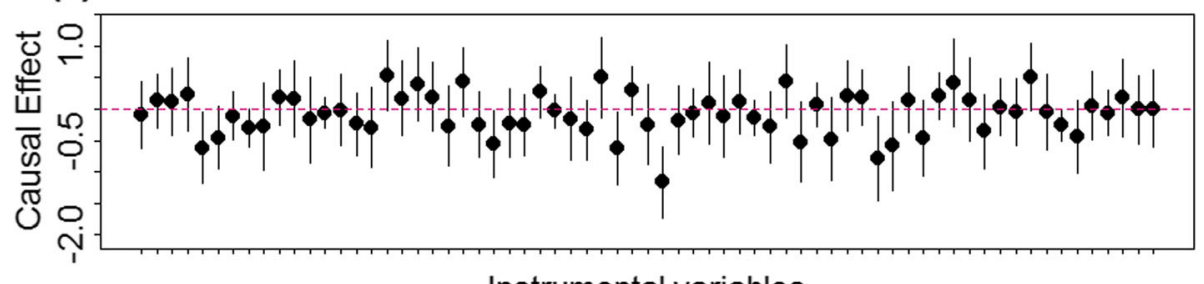

Instrumental variables

Fig. 2 a Relationship between the effect size estimates on T2D ( $x$-axis) and the effect size estimates on ALS ( $y$-axis) for all SNPs that served as instrumental variables for T2D in the European population. Here, a total of 67 T2D instrumental variables were employed. The $95 \%$ confidence intervals for the estimated SNP effect sizes on ALS are shown as vertical black lines, while the $95 \%$ confidence intervals for the estimated SNP effect sizes on T2D are shown as horizontal black lines. The slope of fitted lines represents the estimated causal effect of T2D on ALS obtained using either the IVW method (red lines) or the MR-Egger regression (blue lines). Two possible SNP outliers (i.e., rs7729395 and rs1758632) are highlighted in green. b Funnel plot displays individual causal effect estimates for T2D on ALS in the European population. The dots represent the estimated causal effect for each instrumental variable. The vertical dotted red line represents the estimated causal effect obtained using all instrumental variables. A possible outlier (i.e., rs 1758632) is highlighted in green. c Forest plot for individual causal effect estimate

fasting glucose $(\mathrm{mmol} / \mathrm{L})$, fasting insulin $(\mathrm{pmol} / \mathrm{L})$, and HbA1c (\%) on ALS are estimated to 1.00 (95\% CI 0.871.16, $p=0.931$ ), 1.09 (95\% CI 0.90-1.31, $p=0.370$ ), 0.78 (95\% CI $0.49-1.24, p=0.286$ ), and 0.97 (95\% CI $0.67-$ $1.39, p=0.861$ ), respectively. Details of the unit are further provided in Additional file 3.

\section{Sensitivity analyses to validate the estimated causal effect of T2D on ALS}

We performed extensive sensitivity analyses to validate the causal association between T2D and ALS. Due to the nonsignificant associations between T2D-related glycemic/anthropometric traits and ALS, we only summarized these results in Table 3 and Additional file 1: Figure S3, but did not pursue any of these T2D-related traits further. In addition, as explained in the previous section, in addition to using the final set of $67 \mathrm{~T} 2 \mathrm{D}$ instrumental variables, we also performed sensitivity analyses using all 139 T2D instrument variables (results shown in Additional file 1: Figure S4). The MR-PRESSO analysis shows that one SNP seems to be an instrumental outlier at the nominal significance level of 0.05 (rs1758632, which was located within gene UBAP2 and showed a large effect size on ALS with beta $=0.057$ and $\mathrm{se}=0.014$ ). Removing this instrumental variable does not lead to a substantial change of the causal effect $(\mathrm{OR}=0.95$, 95\% CI $0.89-1.00, p=0.060)$. The LOO results suggest that no single instrumental variable can influence the estimated causal effect (Additional file 1: Table S11). For example, after removing rs7729395 (located within gene $P A M)$, which showed the greatest effect size on both T2D (beta $=0.137$, se $=0.016$ ) and ALS

Table 3 Summary of the causal effects of T2D and T2D-related glycemic/anthropometric traits on ALS with various MR methods

\begin{tabular}{lllll}
\hline Exposures (unit) & $\begin{array}{l}\text { Random- } \\
\text { effects IMW }\end{array}$ & $\begin{array}{l}\text { Weighted } \\
\text { median }\end{array}$ & & MR-Egger \\
\cline { 4 - 5 } & $0.93(0.87-1.00)$ & $0.94(0.86-1.03)$ & $0.90(0.72-1.14)$ & $0.002(0.786)$ \\
T2D & $1.00(0.87-1.16)$ & $0.93(0.77-1.13)$ & $0.80(0.56-1.15)$ & $0.007(0.170)$ \\
BMI $\left(4.8 \mathrm{~kg} / \mathrm{m}^{2}\right)$ & $1.09(0.90-1.31)$ & $1.08(0.98-1.43)$ & $1.10(0.97-1.57)$ & $0.001(0.852)$ \\
Fasting glucose $(\mathrm{mmol} / \mathrm{L})$ & $0.78(0.49-1.24)$ & $0.66(0.33-1.31)$ & $1.91(0.06-61.7)$ & $-0.015(0.615)$ \\
Fasting insulin (pmol/L) & $0.97(0.67-1.39)$ & $0.85(0.55-1.32)$ & $0.95(0.47-1.91)$ & $0.001(0.875)$ \\
HbA1c (\%) & & & &
\end{tabular}


(beta $=-0.077$, se $=0.037)$ among all instruments, the OR of T2D on ALS is estimated to be 0.94 (95\% CI $0.89-1.00, p=0.039$ ). Removing both rs7729395 and rs1758632 leads to a slightly conservative estimate with the OR of T2D on ALS estimated to be 0.95 (95\% CI 0.90-1.01, $p=0.098$ ). Nevertheless, all these estimates are consistent with the causal effect estimated in the previous section using all the available instrumental variables.

The weighted median method also yielded a similar point estimate of causal effect of T2D on ALS (OR = $0.94,95 \%$ CI $0.86-1.03, p=0.159)$. With MR-Egger regression, the OR of T2D on ALS is estimated to be 0.90 (95\% CI 0.72-1.14, $p=0.390$ ), which is consistent with the main results. Importantly, the intercept of MR-Egger is not significantly deviated from zero $(0.002,95 \% \mathrm{CI}$ -0.012-0.017, $p=0.768$ ), suggesting that no apparent horizontal pleiotropy was detected. In addition, the funnel plot also displays symmetric pattern of effect size variation around the point estimate (Fig. $2 \mathrm{~b}$ ), again indicating no apparent horizontal pleiotropy. To alleviate further concerns with horizontal pleiotropy, we excluded all the 17 instruments that have possible pleiotropic effects (Additional file 1: Table S7) and found that the resulted causal effect estimate remains largely unchanged
$(\mathrm{OR}=0.93,95 \%$ CI $0.87-0.99, p=0.047)$. When we excluded ten instruments that were associated with BMI and BMI-related traits (e.g., birth weight, height, waist circumference, waist-hip ratio, and weight), we also obtained a similar causal effect estimate $(\mathrm{OR}=0.93,95 \%$ CI $0.88-1.00, p=0.036$ ). Together, these results suggest that horizontal pleiotropy would unlikely bias the estimated causal effect of T2D on ALS. In addition, the causal effect of ALS on T2D is not statistically significant $(\mathrm{OR}=1.03,95 \% \mathrm{CI} 0.94-1.13, p=0.505)$, ruling out the probability of reverse causation.

Finally, we investigated the causal relationship between T2D and ALS in the East Asian population. Due to the much smaller sample size of ALS (Table 1), the estimated causal effect of T2D on ALS in the East Asian population is not statistically significant at the level of 0.05. Specifically, the OR of T2D on ALS is estimated to be 1.17 (95\% CI 0.93-1.47, $p=0.190$ ). After removing two potential outliers (i.e., rs12219514 and rs75536691), however, the causal effect of T2D on ALS becomes stronger and marginally statistically significant ( $\mathrm{OR}=$ 1.28, 95\% CI 0.99-1.62, $p=0.058$ ) (Fig. 3a), suggesting that T2D may increase the risk of ALS in the East Asian population. Note that the positive causal effect of T2D on ALS in the East Asian population is in contrast with

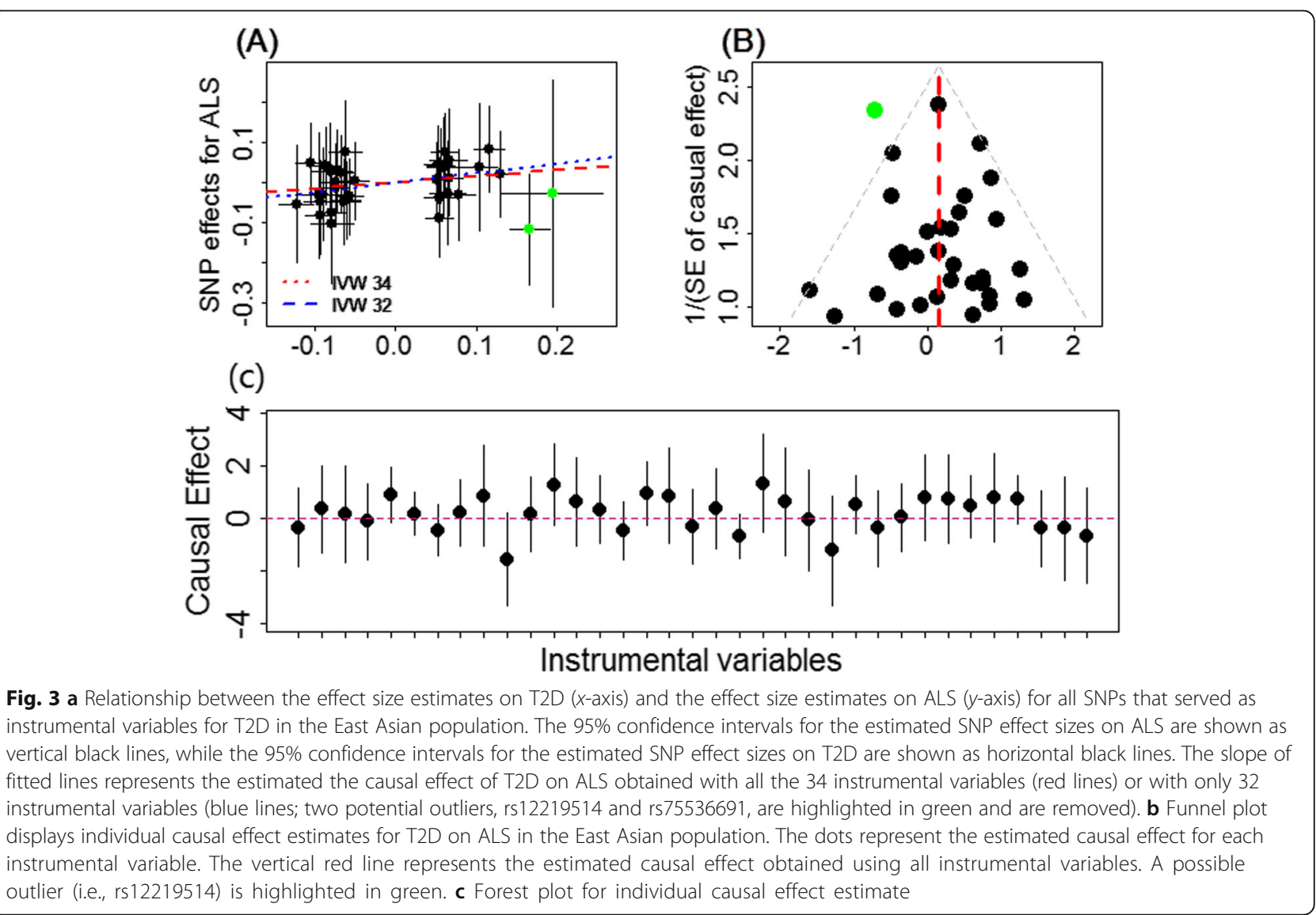


the negative causal effect of T2D on ALS estimated in the European population. Our sensitivity analyses further support the conclusion in the East Asian population. For example, the funnel plot displays a symmetric pattern of effect size variation around the point estimate (Fig. 3b), and the result of MR-Egger regression also rules out the possible influence of horizontal pleiotropy in our analysis (e.g., the MR-Egger intercept is estimated to be 0.021 , with $95 \%$ CI $-0.039-0.081, p=0.488$ ).

\section{Discussion}

\section{Summary of the results of the present study}

It has long been controversial whether T2D is causally protective against ALS or whether ALS is causally protective against T2D $[10,11]$. Here, we have carried out a comprehensive two-sample MR analysis to clarify this controversial issue. To the best of our knowledge, our study is the first exploration that attempts to illuminate the directional causal relationship between T2D and ALS using a genetic approach by leveraging summary statistics from large-scale GWASs. Our results support a neuroprotective role of T2D on ALS in the European population.

As the validity of the MR analysis depends on strong model assumptions, we have carefully selected instrumental variables for T2D in order to satisfy those assumptions. We have conducted extensive sensitivity analyses in the present study to guard against various possible model misspecifications. Given the pervasive pleiotropy among complex traits [73], we attempted to remove instrumental variables with potential pleiotropic effects (Fig. 1 and Additional file 1: Table S7). To quantify the genetic covariance between T2D and ALS, we applied the linkage disequilibrium score regression (LDSC) [74] - a novel approach that can make full use of the genome-wide pleiotropy. With LDSC, consistent with what has been observed in a recent study [52], we found no genetic correlation between T2D and ALS in the European population $\left(R_{\mathrm{g}}=-0.011, \mathrm{se}=0.020, p=\right.$ $0.581)$ or in the East Asian population $\left(R_{\mathrm{g}}=0.051\right.$, se $=$ $0.071, p=0.469)$. The LDSC results suggest a lack of polygenic pleiotropy between T2D and ALS, supporting the issue that pleiotropic instrumental variables unlikely exist in our MR study. These observations are further supported by the distinct pattern of Manhattan plots for T2D and ALS in the two populations (Additional file 1: Figure S5). Therefore, we believe that the employed index SNPs of T2D in our analyses are valid instrumental variables that adequately satisfy the model assumptions required by MR.

One of the advantages of our study is the large-scale GWASs we used, where the large sample sizes allow us to fully establish a credible causal relationship between T2D and ALS in the European population. Importantly, the inferred causal relationship between T2D and ALS is robust with respect to the choice of statistical methods and is carefully validated through various sensitivity analyses and multiple sets of instruments. Overall, our study provides new evidence supporting the causal role of T2D on decreasing the risk of ALS in the European population. Therefore, given that little has been understood about the risk factors for ALS to date [8], our study is an important addition to the line of ALS research and has an important implication for public health. Our results shed light on the pathology of ALS and have the potential to pave the way towards new therapeutic strategies for ALS [75].

\section{Various associations between T2D and ALS in the East Asian and European populations}

Our causal association results are consistent with the association results obtained in several previous observational epidemiological studies (Table 1 and Additional file 1: Table S1). For example, negative associations between T2D and the risk of ALS were consistently observed in the European population [11, 14, 16, 19]. However, we note that the neuroprotective association between T2D and ALS in the European population does not appear to generalize to the East Asian population. Indeed, our MR analysis provides empirically suggestive evidence that T2D might instead increase the risk of ALS in the East Asian population (OR $=1.17$ or 1.28), which is in line with a previous East Asian study (Table 1 and Additional file 1: Table S1) [22].

Given the substantial difference of ALS in terms of clinical features and potential molecular mechanisms between European and East Asian populations, our finding does not come as a surprise. First, the pathophysiology of ALS differs in patients of different ethnicity [27, 63]. It has been well characterized that the risk of developing ALS in East Asia is much lower than that in Europe [76]; the mean age of ALS onset in East Asia is also significantly earlier than that in other countries [77]. Therefore, ALS in the two populations may be heterogenous and influenced by different biological pathways. It is thus possible that T2D affects different biological pathways underlying ALS in different pupations, leading to different observed effects on ALS in the two populations. Second, the genetic architecture underlying ALS may be distinct in the two populations [63, 78, 79]. For example, the expansion of C9orf72-the most common ALS associated gene in the European population-has a much lower frequency in the East Asian ALS patients $(0.30 \%$ vs. $7.00 \%)$. In addition, we found that SNPs located in C9orf72 of T2D patients are significantly positively associated with ALS in the East Asian population (Fig. 4a: $r=0.50,95 \%$ CI $0.41-0.57$, $p=5.41 \mathrm{E}-26)$ but are significantly negatively associated 
(A)

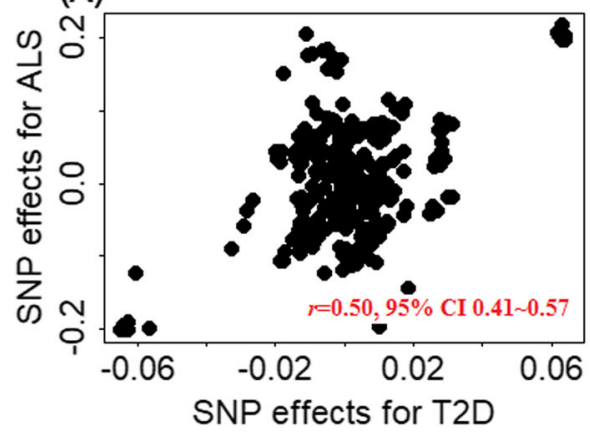

(B)

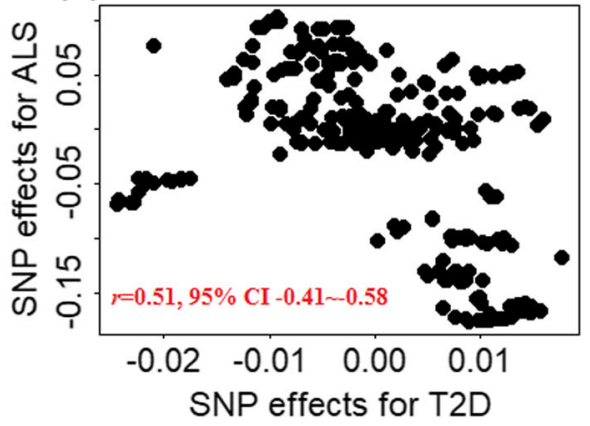

Fig. 4 Pearson's product-moment correlation between the effect size estimates on T2D ( $x$-axis) and the effect size estimates on ALS ( $y$-axis) for all SNPs that were located in gene C9orf72 in the East Asian (a) and European (b) populations. Defined by GENCODE (version 19) [80], a total of 339 SNPs located within C9orf72 (Chr 9: 27,446,544 27,673,864) were included in the two correlation plots

with ALS in the European population (Fig. 4b: $r=0.51$, 95\% CI $-0.41--0.58, p=5.99 \mathrm{E}-27)$. Subsequently, T2D may influence a genetic pathway that has the opposite effects on ALS in the two populations [63, 81, 82]. Indeed, we found that the genetic covariances between T2D and ALS estimated through LDSC analysis also have opposite directions in the two populations: T2D and ALS are negatively genetically correlated in the European population $\left(R_{\mathrm{g}}=-0.011\right)$ but positively genetically correlated in the East Asian population $\left(R_{\mathrm{g}}=\right.$ 0.051). Finally, different genetic architecture, different environment exposure, and ALS disease heterogeneity may interact with each other to lead to different T2D effects on ALS in the European and East Asian populations [14]. Therefore, the biological mechanism underlying the different effects of T2D on ALS across populations can be multifactorial and warrant future investigations.

Certainly, we cannot exclude the possibility that the nonsignificant positive association between T2D and ALS in the East Asian population may be simply due to a lack of power there, as the sample size of ALS in the East Asian population is much smaller compared with that in the European population (1234/2850 vs. 20,806/ 59,804 ) (Table 2). We calculated power using the analytic method developed in [83] (https://cnsgenomics.shinyapps.io/mRnd/), by assuming that the true causal OR of T2D on ALS is 1.20 (or equivalently 0.80) (approximately equal to that estimated in the East Asian population), $\mathrm{PVE}=0.01$ (approximately equal to that estimated in terms of the used instrumental variables), the significance level $\alpha$ is 0.05 , and the proportion of ALS cases is the same as that in the respective ALS GWASs. With this analytic approach, we estimated the statistical power of the two-sample MR detecting such causal effect to be only $9 \%$ in the East Asian population; this is in contrast to an estimated statistical power of $72 \%$ in the European population.
Nevertheless, our findings suggest that a different mechanism may underlie the association between T2D and ALS in the two populations, which is important for understanding the ALS pathology and developing its clinical treatment in different countries. For example, some drug interventions that are effective in the European ALS patients may not necessarily work for East Asian individuals or vice versa. Further investigations on this important issue are warranted in the future.

\section{Other new contributions from the present study}

Besides revealing the neuroprotective role of T2D for ALS in the European population and providing suggestive evidence of increasing the risk of ALS in the East Asian population, our study also, at least in part, solves several previously unanswered questions [75]. First, we demonstrated that, except for T2D itself, no common T2D-related exposures investigated in the present study showed a causal relationship with ALS. These results suggest that those T2D-related exposures might not mediate the influence of T2D on ALS and that T2D is causally protective against ALS via some other unknown pathways. Therefore, intervening fasting glucose and insulin in T2D patients might not impact the risk of ALS or slow its disease progression. Furthermore, previous studies have showed that T2D is positively related to Parkinson's disease $[84,85]$ but is not associated with Alzheimer's disease [57, 86]. Therefore, the lack of association between T2D and Alzheimer's disease, together with the positive association between T2D and Parkinson's disease, imply that the effect of T2D on the motor neurons may be selectively protective and is special for ALS.

\section{Mechanisms underlying the causal associations between T2D and ALS}

We note that the causal relationship between T2D and ALS identified in the present study does not necessarily 
imply that T2D itself directly affects ALS. Instead, pathways associated with T2D may affect the risk of ALS, or, alternatively, T2D may affect ALS through indirectly pathways. Indeed, the mechanisms underlying the causal associations between T2D and ALS may be considerably complex. Several explanations for such causal association exist. For example, it is well known that T2D is associated with higher blood lipids [87], which could resist against the increased energy consumption and hypermetabolism of ALS patients. Therefore, T2D may act through blood lipids to reduce the hypermetabolic damage on the motor neuron system [88], potentially delaying ALS onset and increasing the survival time of ALS patients [12, 89-92]. As another explanation, T2D was recently reported to be associated with higher concentrations of progranulin [93], which could mediate fat-induced insulin resistance and revert mutant TDP-43 (TAR DNA-binding protein 43)-induced axonopathy $[75,94]$. Therefore, T2D may act through TDP-43, which is a well-known risk factor of ALS $[8,95,96]$. As a third explanation, medical treatments and reverted lifestyle (e.g., smoking cessation) for T2D patients may be also beneficial to reduce the risk of ALS.

\section{Some limitations}

Some limitations of this study should be considered. First, similar to other MR studies, we acknowledge that the validity of our MR relies on some crucial modeling assumptions (Additional file 1: Figure S2A) [30, 31, 40], some of which cannot be fully tested for in the framework of two-sample MR. In addition, we also acknowledge that MR cannot fully rule out all the confounders even though MR is less susceptible to reverse causation and confounders compared with other study designs [97]. For example, we cannot completely rule out the indirect role of BMI in the causal relation between T2D and ALS, as it was shown that the nutritional intervention for ALS patients to raise BMI can prolong the survival time and result in the delay of disease progression [90-92] and increasing BMI is directly related to the risk of T2D. Thus, we emphasize that the results generated in the present study should be interpreted with caution, even though we have been extremely careful in selecting instruments to satisfy various model assumptions and have conducted extensive sensitivity analyses to guard against model assumption misspecifications.

Second, as in other previous MR studies, we assumed a linear effect relationship between T2D and ALS in the MR model. While linearity is a first-order approximation of any nonlinear relationship, a simple linearity assumption may not always be reasonable in practice. Indeed, we cannot fully rule out the possibility of nonlinear association between T2D and ALS. In addition, no data on the severity and duration of T2D are available.
Therefore, similar to other MR analyses, we cannot assess the dose-response relationship between T2D and ALS, which is an important aspect of causal inference.

Third, previous studies have shown that the impact of T2D on ALS can be age (and/or gender) dependent [16, 19, 22]. For example, some studies have identified a protective effect of T2D on ALS in old people while identified zero effect or adverse effect in younger ones. As another example, some studies have shown that men may have a higher risk than women in East Asian individuals [98, 99]. Unfortunately, because it is almost impossible to obtain individuallevel data in GWAS due to privacy concerns, we cannot directly estimate the causal effect between T2D and ALS stratified by gender or age groups.

Fourth, the clinical heterogeneity of ALS was recognized in previous studies [100] and some studies have implied that T2D may have a stronger protective effect in bulbar ALS compared with spinal ALS [14]. In the present study, unfortunately, we are unable to further investigate the effect of T2D on sub-phenotypes of ALS due to lack of individual-level information.

Fifth, likely due to the relatively small sample size for ALS cases and the relatively weak exposure effects on the outcome, the statistical power of our MR analysis is limited for certain exposures such as glycemic traits. For example, when the true causal OR is 0.90 (approximately equal to the estimates in the present study), the statistical power to detect a causal effect of fasting insulin (or hemoglobin A1c) on ALS is only $14 \%$ (or $43 \%$ ) with an analytic method proposed in [83]. Note that the calculated power is smaller than that for T2D in the European population because a weaker true OR is assumed here (0.90 vs. 0.80). Certainly, besides the lack of power and the absence of true causal effect, other possible explanations, such as potential unmodeled nonlinear effect, unidentified reverse causality, shared genetic contribution, and uncontrolled confounding effect, may all contribute to the potential failure to detect the association between glycemic traits and ALS. Therefore, larger longitudinal studies with individual-level data in the future are needed to fully establish the causal relationship between those glycemic traits and ALS. These future studies can help elucidate the potential mediating role of glycemic traits in the T2D effects on ALS.

\section{Conclusion}

In conclusion, based on the MR results obtained from large-scale GWAS summary statistics, our study provides new evidence on the causal neuroprotective role of T2D on the risk of ALS in the European population as well as empirically suggestive evidence of increasing risk of T2D on ALS in the East Asian population. 


\section{Supplementary information}

Supplementary information accompanies this paper at https://doi.org/10. 1186/s12916-019-1448-9.

Additional file 1. Supplementary Figures and Tables.

Additional file 2. An Overview of the Mendelian Randomization Method.

Additional file 3. Description of GWAS Data Sets.

\section{Abbreviations}

ALS: Amyotrophic lateral sclerosis; GWAS: Genome-wide association study; IWW: Inverse-variance weighted; LOO: Leave-one-out; MR: Mendelian randomization; MR-PRESSO: Mendelian Randomization Pleiotropy RESidual Sum and Outlier; OR: Odds ratio; PVE: Phenotypic variance explained; SNP: Single-nucleotide polymorphism; T2D: Type 2 diabetes

\section{Acknowledgements}

We thank all the GWAS consortiums for making the summary data publicly available and are grateful to all the investigators and participants who contributed to those studies. We would like to thank the editor, the associate editor, and the reviewers for their constructive comments, which substantially improved our manuscript. We would also like to thank Xinghao Yu and Yixing Gao for their assistance during the revision of this manuscript.

\section{Authors' contributions}

$P Z$, JZ, and $X Z$ conceived the idea for the study. PZ and $X Z$ obtained the genetic data. PZ and TW performed the data analyses. PZ and XZ interpreted the results of the data analyses. All authors wrote the manuscript. All authors read and approved the final manuscript.

\section{Funding}

This study was supported by grants from the National Institutes of Health (R01HG009124 to XZ), the National Science Foundation (DMS1712933 to XZ), the National Natural Science Foundation of Jiangsu (BK20181472 to PZ), the Youth Foundation of Humanity and Social Science funded by the Ministry of Education of China (18YJC910002 to PZ), the General China Postdoctoral Science Foundation (2018M630607 to PZ), the Special China Postdoctoral Science Foundation (2019T120465 to PZ), the Jiangsu QingLan Research Project for Outstanding Young Teachers and the Six-Talent Peaks Project of Jiangsu Province of China (WSN-087 to PZ), the Postdoctoral Science Foundation of Xuzhou Medical University (to PZ), the National Natural Science Foundation of China (81402765 to PZ), the Statistical Science Research Project from the National Bureau of Statistics of China (2014LY112 to PZ), the Priority Academic Program Development of Jiangsu Higher Education Institutions (PAPD) for Xuzhou Medical University (to PZ), and Jiangsu Provincial Key Medical Discipline and the Project of Invigorating Health Care through Science, Technology and Education (ZDXKA2016014 to JZ).

\section{Availability of data and materials}

All data generated or analysed during this study are included in this published article and its supplementary information files.

\section{Ethics approval and consent to participate}

Not applicable.

\section{Consent for publication}

Not applicable.

\section{Competing interests}

The authors declare that they have no competing interests.

\section{Author details}

'Department of Epidemiology and Biostatistics, School of Public Health, Xuzhou Medical University, Xuzhou 221004, Jiangsu, China. ${ }^{2}$ Cancer Institute, Xuzhou Medical University, Xuzhou 221004, Jiangsu, China. ${ }^{3}$ Center of Clinical Oncology, Affiliated Hospital of Xuzhou Medical University, Xuzhou 221004, China. ${ }^{4}$ Jiangsu Center for the Collaboration and Innovation of Cancer Biotherapy, Cancer Institute, Xuzhou Medical University, Xuzhou 221004, Jiangsu, China. ${ }^{5}$ Department of Biostatistics, University of Michigan,
Ann Arbor, MI 48109, USA. ${ }^{6}$ Center for Statistical Genetics, University of Michigan, Ann Arbor, Ml 48109, USA.

Received: 14 March 2019 Accepted: 22 October 2019

Published online: 04 December 2019

\section{References}

1. Kiernan MC, Vucic S, Cheah BC, Turner MR, Eisen A, Hardiman O, Burrell JR, Zoing MC. Amyotrophic lateral sclerosis. Lancet. 2011;377(9769):942-55.

2. Marin B, Boumédiene F, Logroscino G, Couratier P, Babron M-C, Leutenegger AL, Copetti M, Preux P-M, Beghi E. Variation in worldwide incidence of amyotrophic lateral sclerosis: a meta-analysis. Int J Epidemiol. 2017:46(1):57-74.

3. Chio A, Logroscino G, Hardiman O, Swingler R, Mitchell D, Beghi E, Traynor BG, Eurals C. Prognostic factors in ALS: a critical review. Amyotroph Lateral Scler. 2009;10(5-6):310-23.

4. Gladman M, Zinman L. The economic impact of amyotrophic lateral sclerosis: a systematic review. Expert Rev Pharmacoecon Outcomes Res. 2015;15(3):439-50.

5. Larkindale J, Yang W, Hogan PF, Simon CJ, Zhang Y, Jain A, Habeeb-Louks EM, Kennedy A, Cwik VA. Cost of illness for neuromuscular diseases in the United States. Muscle Nerve. 2014:49(3):431-8.

6. Arthur KC, Calvo A, Price TR, Geiger JT, Chiò A, Traynor BJ. Projected increase in amyotrophic lateral sclerosis from 2015 to 2040. Nat Commun. 2016;7:12408.

7. Rowland LP, Shneider NA. Amyotrophic lateral sclerosis. N Engl J Med. 2001; 344(22):1688-700

8. Al-Chalabi A, Hardiman O. The epidemiology of ALS: a conspiracy of genes, environment and time. Nat Rev Neurol. 2013:9(11):617-28.

9. Blacher E, Bashiardes S, Shapiro H, Rothschild D, Mor U, Dori-Bachash M, Kleimeyer C, Moresi C, Harnik Y, Zur M, et al. Potential roles of gut microbiome and metabolites in modulating ALS in mice. Nature. 2019; 572(7770):474-80.

10. Hollinger SK, Okosun IS, Mitchell CS. Antecedent disease and amyotrophic lateral sclerosis: what is protecting whom? Front Neurol. 2016;7:47.

11. Mitchell CS, Hollinger SK, Goswami SD, Polak MA, Lee RH, Glass JD. Antecedent disease is less prevalent in amyotrophic lateral sclerosis. Neurodegener Dis. 2015;15(2):109-13.

12. Jawaid A, Salamone AR, Strutt AM, Murthy SB, Wheaton M, McDowell EJ, Simpson E, Appel SH, York MK, Schulz PE. ALS disease onset may occur later in patients with pre-morbid diabetes mellitus. Eur J Neurol. 2010;17(5):733-9.

13. Armon C, Kurland LT, O'Brien PC, Mulder DW. Antecedent medical diseases in patients with amyotrophic lateral sclerosis. A population-based casecontrolled study in Rochester, Minn, 1925 through 1987. Arch Neurol. 1991; 48(3):283-6.

14. D'Ovidio F, d'Errico A, Carna P, Calvo A, Costa G, Chio A. The role of premorbid diabetes on developing amyotrophic lateral sclerosis. Eur J Neurol. 2018;25(1):164-70.

15. Visser AE, Seelen M, Hulsbergen A, de Graaf J, van der Kooi AJ, Raaphorst J, Veldink $J H$, van den Berg LH. Exploring the fitness hypothesis in ALS: a population-based case-control study of parental cause of death and lifespan. J Neurol Neurosurg Psychiatry. 2017;88(7):550-6.

16. Mariosa D, Kamel F, Bellocco R, Ye W, Fang F. Association between diabetes and amyotrophic lateral sclerosis in Sweden. Eur J Neurol. 2015;22(11):1436-42.

17. Seelen M, van Doormaal PTC, Visser AE, Huisman MHB, Roozekrans MHJ, de Jong SW, van der Kooi AJ, de Visser M, Voermans NC, Veldink JH, et al. Prior medical conditions and the risk of amyotrophic lateral sclerosis. J Neurol. 2014;261(10):1949-56.

18. Turner MR, Goldacre R, Ramagopalan S, Talbot K, Goldacre MJ. Autoimmune disease preceding amyotrophic lateral sclerosis: an epidemiologic study. Neurology. 2013;81(14):1222-5.

19. Kioumourtzoglou M-A, Rotem RS, Seals RM, Gredal O, Hansen J, Weisskopf MG. Diabetes mellitus, obesity, and diagnosis of amyotrophic lateral sclerosis: a population-based study. JAMA Neurol. 2015;72(8):905-11.

20. Moglia C, Calvo A, Canosa A, Bertuzzo D, Cugnasco P, Solero L, Grassano M, Bersano E, Cammarosano S, Manera U. Influence of arterial hypertension, type 2 diabetes and cardiovascular risk factors on ALS outcome: a population-based study. Amyotroph Lateral Scler Frontotemporal Degener. 2017;18(7-8):590-7. 
21. Körner S, Kollewe K, Ilsemann J, Müller-Heine A, Dengler R, Krampfl K, Petri S. Prevalence and prognostic impact of comorbidities in amyotrophic lateral sclerosis. Eur J Neurol. 2012;20(4):647-54.

22. Sun Y, Lu C-J, Chen R-C, Hou W-H, Li C-Y. Risk of amyotrophic lateral sclerosis in patients with diabetes: a nationwide population-based cohort study. J Epidemiol. 2015;25(6):445-51.

23. Vandoorne T, De Bock K, Van Den Bosch L. Energy metabolism in ALS: an underappreciated opportunity? Acta Neuropathol (Berl). 2018;135(4): 489-509.

24. Dupuis L, Pradat P-F, Ludolph AC, Loeffler J-P. Energy metabolism in amyotrophic lateral sclerosis. Lancet Neurol. 2011;10(1):75-82.

25. Harno K, Rissanen A, Palo J. Glucose tolerance in amyotrophic lateral sclerosis. Acta Neurol Scand. 1984:70(6):451-5

26. Pradat PF, Bruneteau G, Gordon PH, Dupuis L, Bonnefont-Rousselot D, Simon D, Salachas F, Corcia P, Frochot V, Lacorte J-M, Jardel C, et al. Impaired glucose tolerance in patients with amyotrophic lateral sclerosis. Amyotroph Lateral Scler. 2010;11(1-2):166-71.

27. Logroscino G. Are diabetes and amyotrophic lateral sclerosis related? Nat Rev Neurol. 2015:11:488-90.

28. Jawaid A, Brown JA, Schulz PE. Diabetes mellitus in amyotrophic lateral sclerosis: Dr Jekyll or Mr Hyde? Eur J Neurol. 2015;22(11):1419-20.

29. Lekoubou A, Matsha TE, Sobngwi E, Kengne AP. Effects of diabetes mellitus on amyotrophic lateral sclerosis: a systematic review. BMC Res Notes. 2014;7:171

30. Paternoster L, Tilling K, Davey Smith G. Genetic epidemiology and Mendelian randomization for informing disease therapeutics: conceptua and methodological challenges. PLoS Genet. 2017;13(10):e1006944.

31. Sleiman PM, Grant SF. Mendelian randomization in the era of genomewide association studies. Clin Chem. 2010:56(5):723-8.

32. He $Y$, Timofeeva $M$, Farrington $S M$, Vaughan-Shaw $P$, Svinti $V$, Walker $M$, Zgaga L, Meng X, Li X, Spiliopoulou A, et al. Exploring causality in the association between circulating 25-hydroxyvitamin D and colorectal cancer risk: a large Mendelian randomisation study. BMC Med. 2018;16(1):142.

33. Zhao JV, Schooling CM. Effect of linoleic acid on ischemic heart disease and its risk factors: a Mendelian randomization study. BMC Med. 2019;17(1):61.

34. Zeng P, Zhou X. Causal association between birth weight and adult diseases: evidence from a Mendelian randomization analysis. Front Genet. 2019;10:618

35. Au Yeung SL, Luo S, Schooling CM. The impact of glycated hemoglobin (HbA1c) on cardiovascular disease risk: a Mendelian randomization study using UK Biobank. Diabetes Care. 2018;41(9):1991.

36. Luo S, Au Yeung SL, Zhao JV, Burgess S, Schooling CM. Association of genetically predicted testosterone with thromboembolism, heart failure, and myocardial infarction: Mendelian randomisation study in UK Biobank. Br Med J. 2019;364:1476

37. Hernán MA, Robins JM. Instruments for causal inference: an epidemiologist's dream? Epidemiology. 2006;17(4):360-72.

38. Xue A, Wu Y, Zhu Z, Zhang F, Kemper KE, Zheng Z, Yengo L, Lloyd-Jones $L R$, Sidorenko J, Wu Y, et al. Genome-wide association analyses identify 143 risk variants and putative regulatory mechanisms for type 2 diabetes. Nat Commun. 2018;9(1):2941

39. Lawlor DA, Harbord RM, Sterne JA, Timpson N, Davey Smith G. Mendelian randomization: using genes as instruments for making causal inferences in epidemiology. Stat Med. 2008;27(8):1133-63.

40. Sheehan NA, Didelez V, Burton PR, Tobin MD. Mendelian randomisation and causal inference in observational epidemiology. PLoS Med. 2008;5(8):e177.

41. Au Yeung SL, Lam HSHS, Schooling CM. Vascular endothelial growth factor and ischemic heart disease risk: a Mendelian randomization study. J Am Heart Assoc. 2017;6(8):e005619.

42. Morris AP, Voight BF, Teslovich TM, Ferreira T, Segrè AV, Steinthorsdottir $V$, Strawbridge RJ, Khan H, Grallert H, Mahajan A, et al. Large-scale association analysis provides insights into the genetic architecture and pathophysiology of type 2 diabetes. Nat Genet. 2012:44(9):981-90.

43. Banda Y, Kvale MN, Hoffmann TJ, Hesselson SE, Ranatunga D, Tang H, Sabatti C, Croen LA, Dispensa BP, Henderson M. Characterizing race/ ethnicity and genetic ancestry for 100,000 subjects in the Genetic Epidemiology Research on Adult Health and Aging (GERA) cohort. Genetics. 2015;200(4):1285-95.

44. Bycroft C, Freeman C, Petkova D, Band G, Elliott LT, Sharp K, Motyer A, Vukcevic D, Delaneau O, O'Connell J, et al. The UK Biobank resource with deep phenotyping and genomic data. Nature. 2018;562(7726):203-9.
45. Zeng $P$, Zhou X. Causal effects of blood lipids on amyotrophic lateral sclerosis: a Mendelian randomization study. Hum Mol Genet. 2019;28(4):688-97.

46. Cragg JG, Donald SG. Testing identifiability and specification in instrumental variable models. Econometric Theory. 1993;9(2):222-40.

47. Burgess S, Small DS, Thompson SG. A review of instrumental variable estimators for Mendelian randomization. Stat Methods Med Res. 2017;26(5): 2333-55.

48. Noyce AJ, Kia DA, Hemani G, Nicolas A, Price TR, De Pablo-Fernandez E, Haycock PC, Lewis PA, Foltynie T, Davey Smith G, et al. Estimating the causal influence of body mass index on risk of Parkinson disease: a Mendelian randomisation study. PLoS Med. 2017;14(6):e1002314.

49. Nicolas A, Kenna KP, Renton AE, Ticozzi N, Faghri F, Chia R, Dominov JA, Kenna BJ, Nalls MA, Keagle P, et al. Genome-wide analyses identify KIF5A as a novel ALS gene. Neuron. 2018;97(6):1268-1283.e1266.

50. Global Lipids Genetics C, Willer CJ, Schmidt EM, Sengupta S, Peloso GM Gustafsson S, Kanoni S, Ganna A, Chen J, Buchkovich ML, et al. Discovery and refinement of loci associated with lipid levels. Nat Genet. 2013:45(11): 1274-83.

51. Kanai M, Akiyama M, Takahashi A, Matoba N, Momozawa Y, Ikeda M, Iwata N, Ikegawa S, Hirata M, Matsuda K, et al. Genetic analysis of quantitative traits in the Japanese population links cell types to complex human diseases. Nat Genet. 2018;50(3):390-400

52. Bandres-Ciga S, Noyce AJ, Hemani G, Nicolas A, Calvo A, Mora G, The IC, The International ALSGC, Tienari PJ, Stone DJ, et al. Shared polygenic risk and causal inferences in amyotrophic lateral sclerosis. Ann Neurol. 2019; 85(4):470-81.

53. Mooradian AD. Dyslipidemia in type 2 diabetes mellitus. Nat Clin Pract Endocrinol Metab. 2009:5:150-9.

54. Lotta LA, Sharp SJ, Burgess S, Perry JRB, Stewart ID, Willems SM, Luan JA, Ardanaz E, Arriola L, Balkau B, et al. Association between low-density lipoprotein cholesterol-lowering genetic variants and risk of type 2 diabetes: a meta-analysis. JAMA. 2016:316(13):1383-91.

55. Yeung CHC, Au Yeung SL, Fong SSM, Schooling CM. Lean mass, grip strength and risk of type 2 diabetes: a bi-directional Mendelian randomisation study. Diabetologia. 2019;62(5):789-99.

56. Censin JC, Nowak C, Cooper N, Bergsten P, Todd JA, Fall T. Childhood adiposity and risk of type 1 diabetes: a Mendelian randomization study. PLoS Med. 2017;14(8):e1002362.

57. Østergaard SD, Mukherjee S, Sharp SJ, Proitsi P, Lotta LA, Day F, Perry JRB, Boehme KL, Walter S, Kauwe JS, et al. Associations between potentially modifiable risk factors and Alzheimer disease: a Mendelian randomization study. PLoS Med. 2015;12(6):e1001841.

58. Tan WY, Yarmolinsky J, Lawlor DA, Timpson NJ. Letter regarding article, "Associations of obesity and circulating insulin and glucose with breast cancer risk: a Mendelian randomization analysis". Int J Epidemiol. 2019; [Epub ahead of print]

59. Holmes MV, Ala-Korpela M, Smith GD. Mendelian randomization in cardiometabolic disease: challenges in evaluating causality. Nat Rev Cardiol. 2017:14(10):577-90.

60. Locke AE, Kahali B, Berndt SI, Justice AE, Pers TH, Day FR, Powell C, Vedantam S, Buchkovich ML, Yang J. Genetic studies of body mass index yield new insights for obesity biology. Nature. 2015;518(7538):197-206.

61. Scott RA, Lagou V, Welch RP, Wheeler E, Montasser ME, Luan JA, Magi R, Strawbridge RJ, Rehnberg E, Gustafsson S, et al. Large-scale association analyses identify new loci influencing glycemic traits and provide insight into the underlying biological pathways. Nat Genet. 2012;44(9):991-1005.

62. Wheeler E, Leong A, Liu C-T, Hivert M-F, Strawbridge RJ, Podmore C, Li M, Yao J, Sim X, Hong J, et al. Impact of common genetic determinants of hemoglobin A1c on type 2 diabetes risk and diagnosis in ancestrally diverse populations: a transethnic genome-wide meta-analysis. PLoS Med. 2017; 14(9):e1002383.

63. Benyamin B, He J, Zhao Q, Gratten J, Garton F, Leo PJ, Liu Z, Mangelsdorf M, Al-Chalabi A, Anderson L. Cross-ethnic meta-analysis identifies association of the GPX3-TNIP1 locus with amyotrophic lateral sclerosis. Nat Commun. 2017:8(1):611.

64. Suzuki K, Akiyama M, Ishigaki K, Kanai M, Hosoe J, Shojima N, Hozawa A, Kadota A, Kuriki K, Naito M, et al. Identification of 28 new susceptibility loci for type 2 diabetes in the Japanese population. Nat Genet. 2019;51(3):379-86.

65. Shim H, Chasman DI, Smith JD, Mora S, Ridker PM, Nickerson DA, Krauss RM Stephens M. A multivariate genome-wide association analysis of 10 LDL 
subfractions, and their response to statin treatment, in 1868 Caucasians. PLoS One. 2015;10(4):e0120758.

66. Hartwig FP, Davey Smith G, Bowden J. Robust inference in summary data Mendelian randomization via the zero modal pleiotropy assumption. Int J Epidemiol. 2017;46(6):1985-98.

67. Bowden J, Del Greco MF, Minelli C, Davey Smith G, Sheehan NA, Thompson JR. Assessing the suitability of summary data for two-sample Mendelian randomization analyses using MR-Egger regression: the role of the $\mathrm{I}^{2}$ statistic. Int J Epidemiol. 2016;45(6):1961-74

68. Yavorska OO, Burgess S. MendelianRandomization: an R package for performing Mendelian randomization analyses using summarized data. Int J Epidemiol. 2017;46(6):1734-9.

69. Verbanck M, Chen C-Y, Neale B, Do R. Detection of widespread horizontal pleiotropy in causal relationships inferred from Mendelian randomization between complex traits and diseases. Nat Genet. 2018;50(5):693-8.

70. Bowden J, Davey Smith G, Haycock PC, Burgess S. Consistent estimation in Mendelian randomization with some invalid instruments using a weighted median estimator. Genet Epidemiol. 2016;40(4):304-14.

71. Burgess S, Thompson SG. Interpreting findings from Mendelian randomization using the MR-Egger method. Eur J Epidemiol. 2017;32(5):377-89.

72. Staley JR, Blackshaw J, Kamat MA, Ellis S, Surendran P, Sun BB, Paul DS, Freitag D, Burgess S, Danesh J. PhenoScanner: a database of human genotype-phenotype associations. Bioinformatics. 2016;32(20):3207-9.

73. Solovieff N, Cotsapas C, Lee PH, Purcell SM, Smoller JW. Pleiotropy in complex traits: challenges and strategies. Nat Rev Genet. 2013;14(7):483-95.

74. Bulik-Sullivan B, Finucane HK, Anttila V, Gusev A, Day FR, Loh P-R, ReproGen C, Psychiatric Genomics C, Genetic Consortium for Anorexia Nervosa of the Wellcome Trust Case Control C, Duncan L, et al. An atlas of genetic correlations across human diseases and traits. Nat Genet. 2015;47(11):1236-41.

75. Jawaid A, Abid A, Schulz PE. Diabetes mellitus and amyotrophic lateral sclerosis: time to bridge the gap between the bench and the bedside. Eur J Neurol. 2018;25(1):3-4.

76. Chiò A, Logroscino G, Traynor BJ, Collins J, Simeone JC, Goldstein LA, White LA. Global epidemiology of amyotrophic lateral sclerosis: a systematic review of the published literature. Neuroepidemiology. 2013;41(2):118-30.

77. Liu MS, Cui LY, Fan DS, Chinese ALSA. Age at onset of amyotrophic lateral sclerosis in China. Acta Neurol Scand. 2014;129(3):163-7.

78. Renton AE, Chiò A, Traynor BJ, Cirulli ET, Lasseigne BN, Petrovski S. State of play in amyotrophic lateral sclerosis genetics. Nat Neurosci. 2014;17(1):17-23.

79. He J, Tang L, Benyamin B, Shah S, Hemani G, Liu R. C9orf72 hexanucleotide repeat expansions in Chinese sporadic amyotrophic lateral sclerosis. Neurobiol Aging. 2015;36(9):2660.e1-8.

80. Harrow J, Frankish A, Gonzalez JM, Tapanari E, Diekhans M, Kokocinski F, Aken BL, Barrell D, Zadissa A, Searle S, et al. GENCODE: the reference human genome annotation for The ENCODE Project. Genome Res. 2012;22(9):1760-74.

81. Ogaki K, Li Y, Atsuta N, Tomiyama H, Funayama M, Watanabe H, Nakamura $\mathrm{R}$, Yoshino H, Yato S, Tamura A, et al. Analysis of C9orf72 repeat expansion in 563 Japanese patients with amyotrophic lateral sclerosis. Neurobiol Aging. 2012;33(10):2527.e2511-6.

82. Chen Y, Lin Z, Chen X, Cao B, Wei Q, Ou R, Zhao B, Song W, Wu Y, Shang H-F. Large C9orf72 repeat expansions are seen in Chinese patients with sporadic amyotrophic lateral sclerosis. Neurobiol Aging. 2016;38:217.e215-22.

83. Brion M-JA, Shakhbazov K, Visscher PM. Calculating statistical power in Mendelian randomization studies. Int J Epidemiol. 2013;42(5):1497-501.

84. Yang Y-W, Hsieh T-F, Li C-I, Liu C-S, Lin W-Y, Chiang J-H, Li T-C, Lin C-C. Increased risk of Parkinson disease with diabetes mellitus in a populationbased study. Medicine (Baltimore). 2017;96(3):e5921.

85. Hu G, Jousilahti P, Bidel S, Antikainen R, Tuomilehto J. Type 2 diabetes and the risk of Parkinson's disease. Diabetes Care. 2007:30(4):842-7.

86. Larsson SC, Traylor M, Malik R, Dichgans M, Burgess S, Markus HS. Modifiable pathways in Alzheimer's disease: Mendelian randomisation analysis. BMJ. 2017;359:55375.

87. Collaborators TGO. Health effects of overweight and obesity in 195 countries over 25 years. N Engl J Med. 2017;377(1):13-27.

88. Bouteloup C, Desport JC, Clavelou P, Guy N, Derumeaux-Burel H, Ferrier A, Couratier P. Hypermetabolism in ALS patients: an early and persistent phenomenon. J Neurol. 2009;256(8):1236-42.

89. Steyn FJ, loannides ZA, van Eijk RPA, Heggie S, Thorpe KA, Ceslis A, Heshmat S, Henders AK, Wray NR, van den Berg LH, et al. Hypermetabolism in ALS is associated with greater functional decline and shorter survival. J Neurol Neurosurg Psychiatry. 2018;89(10):1016-23.
90. Wills A-M, Hubbard J, Macklin EA, Glass J, Tandan R, Simpson EP, Brooks B, Gelinas D, Mitsumoto H, Mozaffar T, et al. Hypercaloric enteral nutrition in patients with amyotrophic lateral sclerosis: a randomised, double-blind, placebo-controlled phase 2 trial. Lancet. 2014:383(9934):2065-72.

91. Mattson MP, Cutler RG, Camandola S. Energy intake and amyotrophic lateral sclerosis. NeuroMolecular Med. 2007:9(1):17-20.

92. Kellogg J, Bottman L, Arra EJ, Selkirk SM, Kozlowski F. Nutrition management methods effective in increasing weight, survival time and functional status in ALS patients: a systematic review. Amyotroph Lateral Scler Frontotemporal Degener. 2018;19(1-2):7-11.

93. Qu H, Deng H, Hu Z. Plasma progranulin concentrations are increased in patients with type 2 diabetes and obesity and correlated with insulin resistance. Mediat Inflamm. 2013;2013:360190.

94. Laird AS, Van Hoecke A, De Muynck L, Timmers M, Van Den Bosch L, Van Damme P, Robberecht W. Progranulin is neurotrophic in vivo and protects against a mutant TDP-43 induced axonopathy. PLoS One. 2010;5(10):e13368.

95. Gasset-Rosa F, Lu S, Yu H, Chen C, Melamed ZE, Guo L, Shorter J, Da Cruz S, Cleveland DW. Cytoplasmic TDP-43 de-mixing independent of stress granules drives inhibition of nuclear import, loss of nuclear TDP-43, and cell death. Neuron. 2019; https://doi.org/10.1016/j.neuron.2019.02.038.

96. Mann JR, Gleixner AM, Mauna JC, Gomes E, DeChellis-Marks MR, Needham PG, Copley KE, Hurtle B, Portz B, Pyles NJ, et al. RNA binding antagonizes neurotoxic phase transitions of TDP-43. Neuron. 2019; https://doi.org/10. 1016/..neuron.2019.01.048.

97. Davey Smith G, Ebrahim S. 'Mendelian randomization': can genetic epidemiology contribute to understanding environmental determinants of disease? Int J Epidemiol. 2003;32(1):1-22.

98. Wei Q, Zheng Z, Guo X, Ou R, Chen X, Huang R, Yang J, Shang H. Association between depression and survival in Chinese amyotrophic lateral sclerosis patients. Neurol Sci. 2016;37(4):557-63.

99. Watanabe H, Atsuta N, Nakamura R, Hirakawa A, Watanabe H, Ito M, Senda J, Katsuno M, Izumi Y, Morita M, Tomiyama $\mathrm{H}$, et al. Factors affecting longitudinal functional decline and survival in amyotrophic lateral sclerosis patients. Amyotroph Lateral Scler Frontotemporal Degener. 2015;16(3-4):230-6.

100. Ravits JM, La Spada AR. ALS motor phenotype heterogeneity, focality, and spread: deconstructing motor neuron degeneration. Neurology. 2009;73(10): $805-11$.

\section{Publisher's Note}

Springer Nature remains neutral with regard to jurisdictional claims in published maps and institutional affiliations.

Ready to submit your research? Choose BMC and benefit from

- fast, convenient online submission

- thorough peer review by experienced researchers in your field

- rapid publication on acceptance

- support for research data, including large and complex data types

- gold Open Access which fosters wider collaboration and increased citations

- maximum visibility for your research: over $100 \mathrm{M}$ website views per year

At BMC, research is always in progress.

Learn more biomedcentral.com/submissions 